Revista Signos 2009, 42(69)

$51-70$

\title{
La negociación temática en la co-construcción del conocimiento realizada por estudiantes universitarios
}

\author{
Paulina Núñez \\ Marcela Oyanedel \\ Pontificia Universidad Católica de Chile \\ Chile
}

Resumen: El siguiente trabajo tiene por objetivo caracterizar, tomando como base el modelo co-constitutivo de Per Linell (1998), la construcción colaborativa del conocimiento disciplinar de estudiantes universitarios cuando estudian en grupo. Se entiende como 'Grupo de estudio' un evento oral orientado a una macro-tarea cuyo propósito central es construir y/o reconstruir conocimiento disciplinar. Los datos demuestran que en su interior se negocian varios tipos de conflicto. En este artículo abordamos la negociación temática que se despliega en los conflictos teóricos; específicamente, analizamos y describimos la dinámica del diálogo al interior de los episodios teóricos. Para ello, el artículo se estructura en dos partes: la primera expone las consideraciones teóricas que sirven de sustento para el trabajo; la segunda presenta los resultados del análisis de los movimientos que llevan a cabo los interactuantes. El estudio ha permitido relevar los movimientos más recurrentes del evento, y perfilar una figura de este que da cuenta de la dinámica interaccional que se desarrolla cuando los estudiantes estudian en grupo.

Palabras Clave: Modelo co-constitutivo, aprendizaje colaborativo, encuentros orales con fines de estudio, conocimiento disciplinar, movimiento.

Recibido:

7-XI-2007

Aceptado: 9-V-2008
Correspondencia: Paulina Núñez (pnunez@puc.cl). Departamento de Ciencias del Lenguaje, Facultad de Letras, Pontificia Universidad Católica de Chile. Avenida Vicuña Mackenna 4860, Santiago, Chile. 


\title{
Thematic negotiation in the co-construction of knowledge as conducted by university students
}

\begin{abstract}
In this paper we characterize, based on Per Linell's (1998) co-constitutive model, the co-construction of disciplinary knowledge as conducted by study groups of undergraduate students. 'Study groups' are herein understood as oral events geared to a macro-task, whose main objective is to construct and re-construct disciplinary knowledge. Findings show that various types of conflict are negotiated within these events. This article addresses thematic negotiation as developed during theoretical conflicts, particularly the emerging dialogic dynamics within theoretical episodes. The paper has been organized into two sections: (1) the theoretical considerations upon which this study is grounded; and (2) an analysis of the participants' movements. Data analyses have helped identify and describe recurrent movements in the event and outline the interactional dynamics among undergraduates while studying in group.
\end{abstract}

Key Words: Co-constitutive model, collaborative learning, oral encounters with study purposes, disciplinary knowledge, movement.

\section{INTRODUCCIÓN}

En este artículo abordamos, desde una perspectiva discursiva e interaccional, la co-construcción de conocimiento disciplinar tal y como se manifiesta en grupos de estudio de estudiantes universitarios chilenos ${ }^{1}$. Como señalan Johnson y Johnson (1999), existe consenso en el mundo académico sobre la valoración positiva que asignan los estudiantes al estudio en grupo como forma de crear conocimiento. Los esfuerzos cooperativos dan como resultado que los participantes reconozcan que todos los integrantes del grupo comparten un destino común, trabajen para el beneficio mutuo de manera que todos obtengan créditos del esfuerzo de cada uno, reconozcan que el desempeño de cada uno es provocado mutuamente por uno mismo y por sus colegas, otorguen poder a los demás, se sientan orgullosos y festejen conjuntamente los logros de cualquier integrante del grupo. Sin embargo, si bien el aprendizaje entre pares -y, en especial, el aprendizaje colaborativo- constituye un foco de atención para los investigadores de la educación (Slavin, 1995; Monereo \& Durán, 2001), su estudio desde una perspectiva lingüístico-discursiva ha sido abordado en menor grado (Mercer, 1997).

Para los propósitos de esta investigación, entendemos por 'Grupo de estudio' (GE) un evento oral, cercano a la conversación informal, pero orientado a una meta o macrotarea (estudiar para una prueba, hacer un trabajo de investigación, construir un ensayo, entre otros), heteroconstruido por dos o más alumnos, simétrico en cuanto a estos, pero no necesariamente en cuanto a roles discursivos y no institucionalizado (Harvey, 2006). El propósito central de la reunión es construir y/o reconstruir conocimiento disciplinar, compartiendo, cotejando, ampliando, complementando y modificando los conocimientos individuales, para lo cual los estudiantes desplie- 
gan estrategias diversas. En este sentido, lo que se busca es la construcción de un conocimiento consensuado validado por el grupo; sin embargo, estos encuentros también satisfacen otras necesidades individuales, entre otras, otorgar un sentido de pertenencia y de identidad grupal y promover la constitución de redes de apoyo.

Por lo anterior, los interactuantes negocian sus intenciones particulares con el objetivo de construir un objeto de discurso, entendido como una interpretación consensuada de la realidad. Este contenido común se alcanza por medio de una dinámica argumentativa que versa sobre conflictos de distinta índole, en un continuum que va desde los contenidos más teóricos (propios de la disciplina) hasta contenidos de la vida cotidiana. Puesto que el fin último es construir conocimiento disciplinar, algunos de los tipos de conflicto más importantes son la negociación de definiciones, la de relaciones entre conceptos, la de principios y procedimientos. La construcción de estos objetos de discurso teórico se desarrolla, principalmente, en secuencias de acciones denominadas 'episodios' por Linell (1998). Al respecto, Núñez y Oyanedel (2006) identifican en este evento tres tipos de episodios: a) aquellos episodios externos a la tarea demandada por el docente de la asignatura, denominados 'laterales' (contar chistes, anécdotas, chismes, etc.), b) aquellos internos a la tarea en los que existe predominio de negociación teórica y c) aquellos también internos a la tarea en los que existe predominio de negociación de procedimientos formales asociados a la tarea: selección de tipografía, de organización textual del trabajo, extensión de este, etc. Por su relevancia en el evento, los dos últimos tipos de episodios reciben el nombre de 'nucleares'.

La negociación temática que se despliega en el episodio teórico es lo que se caracteriza en este estudio. Entendemos por construcción temática la elaboración negociada de un significado unitario que, como decíamos, versa en el evento estudiado tanto sobre conceptos como sobre procedimientos.

En este proceso de negociación, los participantes desarrollan acciones o 'movimientos' discursivos: plantean un problema, apoyan una opinión precedente, refutan una opinión, etc. Estos movimientos se asocian tanto a la etapa del proceso de argumentación (apertura, desarrollo o cierre) como a los roles discursivos que se van construyendo en el paso a paso del diálogo.

\section{Aproximaciones teórico-metodológicas}

Para la consecución de los objetivos planteados, tomamos como base el modelo de comunicación llamado 'co-constitutivo', 'dialógico', en los planteamientos de Linell (1998) y Arundale (1999). El modelo explica la comunicación como un fenómeno dialógico que emerge de la dinámica de la interacción a medida que se van produciendo las diferentes emisiones adyacentes, donde las interpretaciones se limitan y se influencian mutuamente, es decir, se co-construyen. El prefijo 
co- refiere a procesos interactivos y debe entenderse como colaboración, cooperación y coordinación entre los participantes (Koike, 2003).

La perspectiva co-constitutiva incorpora la complejidad del dialogismo conversacional y valoriza la relación entre individuos y estructuras sociales, al punto que aspectos individuales y sociales se consideran inseparables y mutuamente constitutivos (Koike, 2003; Harvey, 2006). En este sentido, la aproximación teórica adoptada posibilita la observación y descripción de secuencias de intervenciones en su contexto. Entre los principios básicos de este modelo se encuentran la 'secuencialidad' y la 'co-construcción'. La 'secuencialidad' es el principio según el cual el significado y la forma de las emisiones (o enunciados) dependen de su posicionamiento, es decir, responden a una emisión previa (o varias de ellas) y proyectan emisiones posibles. Por su parte, la 'co-construcción' corresponde al principio en virtud del cual una emisión individual se construye a partir de la comprensión del enunciado de otro y como respuesta al enunciado de otro. Es decir, las acciones comunicativas son proyectos conjuntos y, por lo tanto, no constituyen actos de habla monológicos.

Una fortaleza del modelo es ofrecer la noción de episodio como unidad semántico-pragmática de análisis, en la que se evidencia una estrecha relación entre marco de participación y estructura del tema. En cuanto a este último, Linell (1998) plantea que su progresión es un proceso dinámico, pues es en el devenir de la interacción que el tema surge, se desarrolla y cambia como parte del dinamismo discursivo.

Puesto que existe una estrecha relación entre marco de participación y estructura del tema, abordar la dinámica de un evento implica metodológicamente analizar tanto la estructura secuencial temática como la estructura de la participación. Es así como, desde esta perspectiva, los temas son entendidos como rasgos inherentes a las actividades de los actores al usar el discurso y los contextos son producto de la dinámica del diálogo de respuestas e iniciativas (tomar iniciativa es una invitación a compartir un tema). Así, los autores crean y mantienen intersubjetividad por medio de la coherencia temática, y la interacción se co-construye a través de una negociación de sentido entre los participantes.

En el estudio realizado, más que el encadenamiento de temas -y por lo tanto de episodios- en el nivel macroestructural, interesa caracterizar la dinámica del diálogo al interior de los episodios.

A partir de esta perspectiva y considerando los propósitos de la investigación, se han considerado como unidades básicas de análisis los episodios y los movimientos.

a) Episodios: Constituyen secuencias discursivas de naturaleza dinámica ligadas en su interior tanto temática -por una trayectoria temática unitaria- como pragmáticamente por una actividad común. Corresponden a un nivel intermedio entre los turnos y el evento total (Linell, 
1998). En este sentido, constituyen unidades que están dadas no solo por el tópico que se desarrolla en su interior, sino también por la forma en que los participantes construyen y organizan la interacción. Los episodios están delimitados formalmente por recursos lingüísticos y/o no lingüísticos (quiebre de redes anafóricas, pausas mayores, cambio en la constelación de interactuantes, gestos, etc.) y en cada uno, como se mencionó anteriormente, es posible identificar, en mayor o menor grado, las fases inicial, de desarrollo y final. La fase inicial corresponde al momento de la instauración del tema del episodio; la fase de desarrollo es la etapa de la negociación propiamente tal del objeto teórico que se está construyendo o reconstruyendo; y la fase final constituye el momento en el que se resuelve positiva o negativamente la negociación, o se suspende.

b) Movimiento: Acción discursiva correspondiente a un segmento textual que permite el progreso de la interacción, por ejemplo, aceptar lo dicho anteriormente, rechazarlo, pedir aclaración, etc. De acuerdo con la postura dialogicista asumida, la naturaleza de un movimiento se identifica por los movimientos que le anteceden y le siguen. El movimiento se actualiza mediante recursos lingüísticos pertenecientes a planos diferentes de organización de la lengua (sintácticos, léxicos, fónicos) o no lingüísticos (kinésicos y proxémicos), recursos que están culturalmente aceptados en la comunidad de práctica concernida (Duarte, 1997).

Dentro de este contexto, es posible concebir la interacción verbal como una constelación de movimientos en torno a un tópico y a una intención común (Gille, 2004).

\section{Método, materiales y procedimientos analíticos}

El corpus de esta investigación está conformado por tres eventos videograbados. Los participantes de los grupos de estudio son alumnos universitarios de diferentes carreras del área de las Ciencias Sociales y Humanas de la Pontificia Universidad Católica de Chile y de la Universidad Andrés Bello.

- En el primer evento (E1), participan cuatro estudiantes que cursan primer semestre de Licenciatura en Letras. La tarea que los motiva a reunirse es estudiar para una prueba de la asignatura Gramática Española II.

- El segundo evento (E2) está conformado por tres estudiantes de cuarto semestre. Cursan la asignatura Metodología de la Investigación de la carrera de Sociología y están preparando un trabajo de investigación.

- En el tercer evento (E3) participan cuatro estudiantes de Psicología de quinto semestre. El objetivo de la reunión es estudiar para una prueba de Psicología Educacional. 
La metodología es fundamentalmente cualitativa, pues pretende estudiar en profundidad ciertos fenómenos discursivos en la interacción misma. Las categorías emergen de la interacción y son interpretadas desde su interior.

Los procedimientos analíticos llevados a cabo para el análisis de cada evento son:

1. Videograbación de los eventos. El primer evento fue videograbado por un ayudante de investigación externo al grupo de estudio; en los eventos 6 y 9 , uno de los participantes fue responsable de la grabación. Un estudio experimental previo había demostrado que ninguna de las dos técnicas de videograbación interfería en la dinámica interna del grupo.

2. Transliteración del corpus con ayuda del programa computacional TRANSANA que, junto a los datos lingüísticos, permite registrar fenómenos proxémicos y kinésicos.

3. Determinación del contexto situacional mediante una grilla SPEAKING (Hymes, 1986) a partir del análisis de las grabaciones y de material etnográfico adicional (encuestas y notas de campo).

4. Segmentación del evento en episodios y clasificación de los mismos según propuesta de Núñez y Oyanedel (2006).

5. Identificación de movimientos recurrentes al interior de cada episodio teórico.

6. Determinación de la funcionalidad de los movimientos para estructurar los episodios teóricos y de su actualización lingüística.

En tanto secuencias textuales, los episodios son delimitados mediante diversos recursos formales, algunos de los cuales dan cuenta de su coherencia temática (el episodio constituye un solo tema a nivel global) y otros, de su coherencia pragmática (las microacciones confluyen en una sola unidad accional).

En cuanto a la consideración del episodio como unidad temática, se identificaron recursos formales como: instauración léxica de un tema y mantención de una red léxica asociada a este tema; marcas de progresión desencadenada a partir de la instauración léxica; uso de conectores textuales que den cuenta de las relaciones interproposicionales; quiebre de las redes anafóricas internas; pausas mayores de inicio y de término; expresiones metadiscursivas.

En cuanto a recursos para delimitar cada episodio como unidad pragmática, se consideró el uso de marcadores discursivos, de deícticos, de formas verbales como imperativo y condicional, homogeneidad en la constelación de interactuantes y expresiones metadiscursivas, pausas mayores y, finalmente, la interpretación -por medio de la videograbación- de gestos y cambios proxémicos. 


\section{Resultados}

El análisis de la negociación temática -entendida, como decíamos, como una sucesión de movimientos en torno a un objeto discursivo, en este caso teórico- ha permitido identificar los siguientes movimientos:

1. PROBL Movimiento de Introducir una interrogación problemática

2. PROP Movimiento de Proponer un problema, su solución o una acción

3. ACEPT Movimiento de Aceptar una opinión (con o sin argumentación)

4. RECH Movimiento de Rechazar una opinión (con o sin argumentación)

5. OBJEC Movimiento de Objetar una opinión

6. ACLAR Movimiento de Aclarar una proposición

7. SOLCONF Movimiento de Solicitar confirmación

8. CONF Movimiento de Confirmar una petición

9. SOLINF Movimiento de Solicitar información temática o pragmática

10. SOLOP Movimiento de Solicitar una opinión

11. DARINF Movimiento de Dar información

12. SUSPJ Movimiento de Suspender el juicio

13. RECT Movimiento de Rectificar una aseveración propia o ajena

14. RESPROBL Movimiento de Resolver un problema

15. SUSPROBL Movimiento de Suspender el problema

Los movimientos del listado anterior se diferencian entre sí por la presencia o la ausencia de 6 rasgos binarios:

[+/- Acuerdo] Expresa/No expresa acuerdo con una opinión

[+/- Información] Introduce/No introduce nueva información a una opinión

[+/- Postura] Implica/No implica toma de postura frente a una opinión

[+/- Tema] Introduce/No introduce nuevo tema en la conversación

[+/- Orientación] Se orienta/No se orienta al(los) interactuantes

[+/- Reacción] Reacciona/No reacciona a un movimiento anterior de otro participante.

A continuación, se presenta una matriz con la caracterización de los movimientos según los rasgos recién enunciados. Luego se describen los movimientos detectados, distribuidos según las fases del episodio en que tienden a aparecer mayoritariamente. 
Tabla 1. Rasgos de los movimientos en el evento Grupo de estudio.

\begin{tabular}{|l|c|c|c|c|c|c|}
\hline MOVIMIENTO & ACUERDO & INFORMACIÓN & POSTURA & TEMA & ORIENTACIÓN & REACCIÓN \\
\hline PROBL & 0 & - & $+/-$ & $+/-$ & + & - \\
\hline PROP & 0 & 0 & + & $+/-$ & + & - \\
\hline ACEPT & + & $+/-$ & + & - & + & + \\
\hline RECH & - & $+/-$ & + & - & + & + \\
\hline OBJEC & - & + & + & - & + & + \\
\hline ACLAR & 0 & + & $+/-$ & - & + & + \\
\hline SOLCONF & 0 & + & - & - & + & $+/-$ \\
\hline CONF & + & - & + & - & + & + \\
\hline SOLINF & 0 & - & - & - & + & - \\
\hline SOLOP & 0 & - & - & - & + & - \\
\hline DARINF & 0 & + & $+/-$ & - & + & + \\
\hline SUSPJ & 0 & - & 0 & - & $+/-$ & + \\
\hline RECT & - & + & + & - & + & + \\
\hline RESPROBL & + & - & + & - & + & + \\
\hline SUSPROBL & + & - & 0 & - & + & + \\
\hline
\end{tabular}

\subsection{Fase inicial}

La fase inicial, como se mencionó anteriormente, corresponde al momento de la instauración del tema del episodio. Los movimientos para desencadenar secuencias de desarrollo temático no son exclusivos de esta fase y se presentan recurrentemente en el evento estudiado.

El primer movimiento, 'Introducir una interrogación problemática' (PROBL), tiene como función principal iniciar un episodio teórico a través de la instauración de un tema conflictivo. Por lo general, responde a interrogantes como: ¿qué es?, ¿cómo es?, ¿cuál es?, etc., como se muestra en el ejemplo (1):

(1) E: cuál es el complemento directo $\uparrow$

A: mmm / el nombre $\uparrow$ o el...

E: [el nombre $\uparrow$

A: a ver / mono // ensayémoslo

E: ah... bueno (RISAS)

E1EpT4

La interrogante respecto del complemento directo desencadena una secuencia de movimientos en la que se van posicionando los distintos participantes. 
A continuación se presentan los rasgos que caracterizan este movimiento:

Tabla 2. Movimiento de Introducir una interrogación problemática.

\begin{tabular}{|c|c|c|c|c|c|c|}
\hline MOVIMIENTO & ACUERDO & INFORMACIÓN & POSTURA & TEMA & ORIENTACIÓN & REACCIÓN \\
\hline PROBL & 0 & - & $+/-$ & $+/-$ & + & - \\
\hline
\end{tabular}

Como se muestra en la Tabla 2, se trata de un movimiento neutro en términos de acuerdo, dado que aún los interactuantes no dan inicio a la interacción propiamente tal, y negativo en tanto información. Respecto del rasgo postura, el interactuante puede manifestar o no su posición frente al nuevo objeto temático, en el caso de que este sea planteado, y puede introducir tema nuevo. Este movimiento es aloorientado, pues con él un interlocutor incita a otros a compartir su conflicto intelectual, y es también iniciativo, en la medida que da comienzo al desarrollo de un nuevo conflicto teórico.

Este movimiento tiende a realizarse por medio de una pregunta directa (ejemplo 1) o por medio de una expresión metadiscursiva, como se observa en el ejemplo (2):

(2) A: pero / una pregunta

M: mmm... pregunta

E1EpT7

Otro de los movimientos detectados en los episodios teóricos que sirve, entre otras funciones, frecuentemente para iniciar episodio, es aquel en que uno de los participantes realiza una sugerencia o proposición, el movimiento de 'Proponer un tema-acción' (con o sin argumentación) (PROP). Tal es el caso del ejemplo (1) expuesto anteriormente, en el que Ema cuestiona la respuesta dada por Ana respecto de cuál es el complemento directo en una oración, y esta sugiere al grupo de compañeros que conforman el evento ensayar el ejercicio gráficamente:

E: cuál es el complemento directo $\uparrow$

A: $\mathrm{mmm} /$ el nombre $\uparrow$ o el...

E: [el nombre $\uparrow$

A: a ver / mono // ensayémoslo

E: ah... bueno (RISAS)

E1EpT6

Los rasgos de este movimiento se presentan en la siguiente Tabla:

Tabla 3. Movimiento de Proponer un tema o acción.

\begin{tabular}{|c|c|c|c|c|c|c|}
\hline MOVIMIENTO & ACUERDO & INFORMACIÓN & POSTURA & TEMA & ORIENTACIÓN & REACCIÓN \\
\hline PROP & 0 & 0 & + & $+/-$ & + & - \\
\hline
\end{tabular}


Como se puede apreciar, el movimiento de proposición comparte gran parte de los rasgos del movimiento PROBL. Sin embargo, difiere de este al tratarse de un movimiento en el que el interlocutor expresa implícita o explícitamente su postura, esto es, su deseo de que sea acogida su proposición.

Se destaca la actualización frecuente de dos recursos lingüísticos para llevar a cabo este movimiento: la pregunta encabezada por la subjunción 'si' ('y si') y la forma imperativa de $1^{\text {a }}$ persona, con claro valor incitativo, como muestran los ejemplos (3 y 4 ):

(3) C: y si le ponemos confirmación de la teoría más que una revisión $\uparrow$ A: buen punto / y por qué yo los tenía separados $\uparrow \quad$ E6EpT4

(4) C: yo le puse... claro bibliografía // es que / dónde quedaba el análisis de datos $\uparrow$ dónde... en qué categoría lo encajábamos $\uparrow$ en bibliografía $\uparrow$

A: buen punto / no pusimos eso / $\mathrm{mm} /$ pongámosles análisis estadístico y de datos / no个

E6EpT1

En algunos casos, el enunciado propositivo es reforzado mediante una justificación argumentativa en general encabezada por el conector 'porque', lo que pone en evidencia una actitud colaborativa de los interactuantes para co-construir un conocimiento consensuado, como se puede ver en (5):

(5) C: y también / no puede ser pariente $\uparrow$ porque es un mismo grupo

E6EpT7

\subsection{Fase de desarrollo temático}

En cuanto a los movimientos que participan en el desarrollo de la dinámica argumentativa una vez instaurado el conflicto teórico, estos pueden disponerse en un continuum en cuyos extremos se encuentran, por un lado, el acuerdo pleno de un interactuante respecto de una opinión o proposición anteriormente emitida y, por otro lado, su rechazo total. Entre ambos extremos, es posible identificar una serie de acciones argumentativas que participan en la negociación y la hacen avanzar, y cuyos rasgos se acercan más a uno o a otro extremo.

Hemos denominado ACEPT aquel movimiento de 'Aceptar una opinión' o proposición. El análisis ha permitido identificar que este movimiento puede darse con o sin información que apoye la posición de aceptación del interlocutor. La siguiente Tabla muestra los rasgos que lo caracterizan: 
Tabla 4. Movimiento de Aceptar una opinión.

\begin{tabular}{|c|c|c|c|c|c|c|}
\hline MOVIMIENTO & ACUERDO & INFORMACIÓN & POSTURA & TEMA & ORIENTACIÓN & REACCIÓN \\
\hline ACEPT & + & $+/-$ & + & - & + & + \\
\hline
\end{tabular}

En cuanto a su realización lingüística, se trata de un movimiento realizado frecuentemente mediante repetición (ejemplo 6), expresión metacognitiva (ejemplo 7), marcador discursivo (ejemplo 8) o completación oracional (ejemplo 9), como se muestra a continuación:

(6) C: Joaquín Lavín ganó las elecciones municipales tal año, y describe eso y lo ocupa como argumento en lo que está exponiendo

A: bibliografía

B: bibliografía

C: bibliografía no más

B: bibliografía porque él está recurriendo a una serie de datos de alguna parte

E6EpT1

(7) C: ya po / entonces la repetición está de más / está de más esa categoría

A: repetición de una teoría

C: porque tú decís claro / repetición de una teoría es...

A: en el fondo no aporta conocimiento / o sea / puede ser que aporte conocimiento pero no conocimiento así como...

E6EpT4

(8) B: los negros son una etnia / cierto

C: sí

A: sí po

E6EpT9

(9) C: veamos la definición de cohorte de nuevo

$B$ : es que sale como grupo etario

A: grupo... sí

C: [sale puro grupo etario

B: o sea / no sale puro // es grupo etario / pero todos los ejemplos... todo lo que habla es como

A: generalizado

E6EpT2 
En los casos en que el enunciado de aceptación se acompaña con información que refuerza la postura, una forma de realización recurrente es el uso de marcador discursivo, seguido del apoyo propiamente tal:

(10) J: por ejemplo / envié una carta a Juan // le envié una carta

S: claro / no podí decir lo envié a Juan

E1EpT2

En el extremo opuesto al movimiento de aceptación se encuentra el movimiento de 'Rechazar una opinión' (RECH). Al igual que su movimiento contrario, puede manifestarse con o sin información de apoyo. En el siguiente ejemplo, se aprecia cómo el interactuante A rechaza en forma tajante, sin razones que sustenten su opinión, la proposición de S:

(11) S: ya // núcleo verbo / primera persona singular

A: no

M: plural

S: bah... del plural

E1EpT3

Sus rasgos se presentan en la siguiente Tabla:

Tabla 5. Movimiento de Rechazar una opinión.

\begin{tabular}{|c|c|c|c|c|c|c|}
\hline MoVIMIENTO & ACUERdO & INFORMACIÓN & POSTURA & TEMA & ORIENTACIÓN & REACCIÓN \\
\hline RECH & - & $+/-$ & + & - & + & + \\
\hline
\end{tabular}

Al interior del continuum de la dinámica argumentativa, entre los movimientos de aceptación y de rechazo total, se disponen movimientos de variada índole.

Cercano al movimiento de rechazo se encuentra el movimiento de 'Objetar una Opinión' (OBJEC). La objeción a lo dicho por el interlocutor anterior es menos categórica que el movimiento de rechazo descrito anteriormente, puesto que no invalida la proposición contenida en este, sino más bien plantea uno o más obstáculos, los que generalmente desencadenan movimientos de aclaración adicionales:

(12) C: sí yo creo que es cohorte

B: es que es como comparación

E6EpT2 
La Tabla 6 contiene los rasgos distintivos de este movimiento:

Tabla 6. Movimiento de Objetar una opinión.

\begin{tabular}{|c|c|c|c|c|c|c|}
\hline MOVIMIENTO & ACUERDO & INFORMACIÓN & POSTURA & TEMA & ORIENTACIÓN & REACCIÓN \\
\hline OBJEC & - & + & + & - & + & + \\
\hline
\end{tabular}

Otro movimiento interno es el movimiento de 'Aclarar una opinión', proposición o problematización (ACLAR). Se actualiza frecuentemente por medio de una reformulación, con el marcador discursivo correspondiente. Así lo demuestra el siguiente ejemplo, donde los interactuantes discuten sobre la extensión de un complemento directo:

(13) C: pero el invitado sería juan / no a juan // lo invitado

J: [lo

C: o sea / si yo invitaré a juan

E1EpT8

Tabla 7. Movimiento de Aclarar una proposición.

\begin{tabular}{|c|c|c|c|c|c|c|}
\hline MOVIMIENTO & ACUERDO & INFORMACIÓN & POSTURA & TEMA & ORIENTACIÓN & REACCIÓN \\
\hline ACLAR & 0 & + & $+/-$ & - & + & + \\
\hline
\end{tabular}

Un tercer movimiento al interior del continuum es el movimiento de 'Solicitar confirmación' (SOLCONF), movimiento colaborativo por el cual un participante pide, a otro u otros, confirmación a su proposición, sea esta temática o pragmática:

(14) B: oye / casi toda es tendencia $\uparrow /$ o no $\uparrow$

E6EpT2

Los rasgos que caracterizan este movimiento se presentan en la Tabla 8:

Tabla 8. Movimiento de Solicitar confirmación.

\begin{tabular}{|c|c|c|c|c|c|c|}
\hline MOVIMIENTO & ACUERDO & INFORMACIÓN & POSTURA & TEMA & ORIENTACIÓN & REACCIÓN \\
\hline SOLCONF & 0 & + & - & - & + & $+/-$ \\
\hline
\end{tabular}

Como se puede apreciar, en este movimiento se manifiesta fuertemente el valor interaccional del encuentro Grupo de Estudio. No hay una posición de acuerdo o desacuerdo por parte del participante, sino más bien una apelación al otro en búsqueda de un consenso, lo que apunta al fenómeno de la construcción del conocimiento disciplinar como un proceso de co-construcción de aportes y acuerdos sucesivos. Este movimiento se realiza frecuentemente después de un 
movimiento de proposición, al interior de un mismo turno.

Por lo general, a este movimiento le sigue, como respuesta, el movimiento de 'Confirmar una petición' (CONF), cuyos rasgos se muestran en la siguiente Tabla:

Tabla 9. Movimiento de Confirmar una petición.

\begin{tabular}{|c|c|c|c|c|c|c|}
\hline MOVIMIENTO & ACUERdo & INFORMACIÓN & POSTURA & TEMA & ORIENTACIÓN & REACCIÓN \\
\hline CONF & + & - & + & - & + & + \\
\hline
\end{tabular}

Este movimiento de confirmar presenta acuerdo temático y, al mismo tiempo, refuerza las relaciones interpersonales y la consolidación del grupo ('estamos avanzando'). Su actualización lingüística es, en muchas ocasiones, un marcador pragmático ('claro', 'por supuesto', 'si po'). Este movimiento es cercano al movimiento de aceptación, pero no presenta información nueva de apoyo -que el movimiento de aceptación puede presentar- y es absolutamente reactivo al movimiento precedente SOLCONF.

Paralelamente a movimientos que se caracterizan por su fuerte componente argumentativointeractivo, como los dos anteriormente descritos, existen movimientos que apuntan más al desarrollo del tema en sí, como ocurre con el movimiento de 'Solicitar información' (SOLINF), cuyos rasgos se presentan a continuación:

Tabla 10. Movimiento de Solicitar información.

\begin{tabular}{|c|c|c|c|c|c|c|}
\hline MOVIMIENTO & ACUERdO & INFORMACIÓN & POSTURA & TEMA & ORIENTACIÓN & REACCIÓN \\
\hline SOLINF & 0 & - & - & - & + & - \\
\hline
\end{tabular}

Como se puede apreciar, este movimiento se caracteriza por solicitar un conocimiento declarativo. Sin embargo, el análisis ha permitido también identificar un tipo de petición diferente a este. Se trata de la solicitud por parte de un participante a otro u otros de una opinión-postura respecto de un contenido particular. Este nuevo movimiento es el movimiento de Solicitar opinión:

Tabla 11. Movimiento de Solicitar opinión.

\begin{tabular}{|c|c|c|c|c|c|c|}
\hline MOVIMIENTO & ACUERDO & INFORMACIÓN & POSTURA & TEMA & ORIENTACIÓN & REACCIÓN \\
\hline SOLOP & 0 & - & - & - & + & - \\
\hline
\end{tabular}

Como se demuestra en la Tabla anterior, este movimiento es eminentemente pragmático y aloorientado, puesto que el participante -más que defender una posición o pedir información- 
intenta incluir ostensivamente al otro en la búsqueda de consenso, como se ilustra en el ejemplo (15):

$$
\text { C: no sé qué pensái tú / consuelo E6EpT1 }
$$

La fuerza de la alocución -dada en el ejemplo anterior por el deíctico personal 'tú' y por el vocativo -y en otros casos por formas imperativas del verbo- obliga al interlocutor a responder asumiendo, idealmente, postura, o a suspender marcadamente el juicio, como más adelante se señalará.

Los movimientos de solicitar información y de solicitar opinión pueden desencadenar, siempre y cuando el interlocutor reaccione como se espera, un movimiento reactivo de aporte informativo, que hemos denominado movimiento de 'Dar información nueva' (DARINF).

Tabla 12. Movimiento de Dar información nueva.

\begin{tabular}{|c|c|c|c|c|c|c|}
\hline MOVIMIENTO & ACUERDO & INFORMACIÓN & POSTURA & TEMA & ORIENTACIÓN & REACCIÓN \\
\hline DARINF & 0 & + & $+/-$ & - & + & + \\
\hline
\end{tabular}

Cuando el interlocutor realiza este movimiento, se compromete en diferentes grados con la información que entrega; de allí que el rasgo postura presente los valores de presencia y ausencia $(+/-)$.

No siempre la respuesta a los movimientos SOLINF Y SOLOP posee el valor informativo solicitado. En ciertos casos, el participante realiza un movimiento de 'Suspender el juicio' (SUSPJ) y, en una estrategia de poder, invita al interlocutor que ha solicitado información nueva a especificar su petición. De esta manera, se dilata la entrega de información.

En la siguiente Tabla, presentamos los rasgos de este movimiento:

Tabla 13. Movimiento de Suspender el juicio.

\begin{tabular}{|c|c|c|c|c|c|c|}
\hline MOVIMIENTO & ACUERDO & INFORMACIÓN & POSTURA & TEMA & ORIENTACIÓN & REACCIÓN \\
\hline SUSPJ & 0 & - & 0 & - & $+/-$ & + \\
\hline
\end{tabular}

Como se puede apreciar, esta acción argumentativa no posee valor informativo alguno en términos de contenido temático, pero sí respecto del procesamiento de la información, pues expresa la actitud reflexiva que los participantes asumen como propia en la co-construcción del conocimiento disciplinar. Los siguientes ejemplos demuestran claramente esta actitud. En el primero 
de ellos (16) hay aloorientación, como una reacción ante lo propuesto por otro participante. En el segundo (19), por su parte, existe autoorientación, en el sentido de suspender la confirmación de algo dicho por un mismo participante. En ambos casos, se da cuenta del valor metacognitivo de este movimiento.

(16) B: un panel ocupa encuesta / entrevista y estadística // o no $\uparrow$

A: depende / lo que tú quieras

E6EpT8

(17) C: $\mathrm{mmm}$

E6EpT6

Así como un participante puede suspender la reacción ante un enunciado recién emitido, puede también reaccionar inmediatamente rectificando lo dicho por otro o por él mismo (movimiento de 'Rectificar' - RECT). Es interesante destacar que este movimiento da cuenta, una vez más, del hecho de que la búsqueda de consenso no implica necesariamente un permanente acuerdo; también es necesario estar atento para expresar en el momento oportuno el desacuerdo, siempre condicionado a una actitud no confrontacional, sino cooperativa.

Entre los rasgos que definen este movimiento destacan la entrega de información nueva y la toma de postura. Al igual que otros movimientos, puede ser alo o autoorientado, como se observa en la Tabla 14 y en el ejemplo (18):

Tabla 14. Movimiento de Rectificar una aseveración propia o ajena.

\begin{tabular}{|c|c|c|c|c|c|c|}
\hline MOVIMIENTO & ACUERDO & INFORMACIÓN & POSTURA & TEMA & ORIENTACIÓN & REACCIÓN \\
\hline RECT & - & + & + & - & $+/-$ & + \\
\hline
\end{tabular}

(18) C: veamos la definición de cohorte de nuevo

$B$ : es que sale como grupo etario

A: grupo... sí

C: sale puro grupo etario

B: o sea / no sale puro // es grupo etario / pero todos los ejemplos... todo lo que habla es como

A: generalizado

E6EpT2 


\subsection{Fase final}

Se tiende a pensar que en la fase final -o momento de término del episodio teórico- se resuelve positivamente el problema que está en discusión, es decir, se logra un consenso total de los participantes. Sin embargo, el análisis del corpus ha demostrado que, en ciertos casos y por diversas razones, los participantes deciden dar término a un episodio sin llegar necesariamente a un acuerdo. Entre otras razones, puede ocurrir que la discusión se dilate más de lo necesario y por lo tanto se postergue con la intención de retomarse; se complejice a tal punto la discusión que abra nuevos problemas teóricos, es decir, nuevos episodios; se interrumpa el desarrollo del episodio por acción de factores externos y no se retome. Cuando el episodio se cierra con acuerdo temático, el movimiento se ha denominado movimiento de 'Resolver el problema' (RESPROBL) y cuando el cierre ocurre por las otras razones antes mencionadas nos encontramos frente al movimiento de 'Suspender el problema' (SUSPROBL).

Como se demuestra en las siguientes tablas, ambos movimientos solo difieren en el rasgo postura. El movimiento RESPROBL es una acción discursiva que conlleva una posición de un participante respecto del objeto teórico, posición que es aceptada por el resto de los miembros que constituyen el grupo de estudio. Discursivamente se manifiesta a través de marcadores de cierre textual 'bueno', 'en resumen', 'listo', 'filo', 'ya' o en un juego de marcas personales, como se observa en el ejemplo (19):

(19) A: yo lo pondría como cohorte / porque es una población súper específica / no es una población en general // no son todos los negros...

B: [ya /voy a poner cohorte

C: pongamos cohorte

E6EpT2

Tabla 15. Movimiento de Resolver el problema.

\begin{tabular}{|c|c|c|c|c|c|c|}
\hline MOVIMIENTO & ACUERDO & INFORMACIÓN & POSTURA & TEMA & ORIENTACIÓN & REACCIÓN \\
\hline RESPROBL & + & - & + & - & + & + \\
\hline
\end{tabular}

Contrariamente, en el movimiento de suspender el problema, el rasgo postura no resulta ser pertinente en el episodio teórico. Como se dijo anteriormente, el desarrollo de la negociación es interrumpido antes de alcanzarse un acuerdo temático. Solo se produce el acuerdo pragmático de cerrar el episodio. En este sentido, es importante recordar que los movimientos son acciones que se materializan discursivamente; por lo tanto, si un episodio se suspende por la intervención de un factor externo y sus integrantes no realizan un movimiento perceptible de suspensión -verbal o gestual-, como por ejemplo por la llegada imprevista de una persona no miembro del grupo de estudio, no se hablará de movimiento de suspender el problema. 
Tabla 16. Movimiento de Suspender el problema.

\begin{tabular}{|c|c|c|c|c|c|c|}
\hline MOVIMIENTO & ACUERDO & INFORMACIÓN & POSTURA & TEMA & ORIENTACIÓN & REACCIÓN \\
\hline SUSPROBL & + & - & 0 & - & + & + \\
\hline
\end{tabular}

En síntesis, el trabajo de relevar los movimientos recurrentes del evento Grupo de estudio ha ido perfilando una figura de este tipo de encuentro donde el conflicto constituye un factor central como motor mismo de la dinámica interaccional. La escasa bibliografía sobre el tipo de interacción estudiada confirma esa característica. Los movimientos que abren episodio teórico -fundamentalmente el de problematización- instauran el conflicto. En los movimientos siguientes, los interactuantes toman postura, plantean sus posiciones, negocian, haciendo avanzar el tema y resolviendo paso a paso los conflictos internos que van surgiendo. Por último, en los movimientos que cierran episodio, los interactuantes llegan a un acuerdo, solucionándose el conflicto inicial, o bien se suspende el acuerdo por factores internos o externos.

Paradojalmente, un rasgo común a los movimientos hasta ahora detectados en este primer análisis es su carácter colaborativo. Incluso aquellos movimientos que son descritos con predominancia de rasgos neutros o negativos -como objeción, rectificación o rechazo- se entienden como colaborativos en este evento particular. Su actualización preferente con un apoyo argumentativo que acompaña al núcleo del enunciado, confirma ese valor. En efecto, muchos enunciados presentan una estructura dual, un núcleo que es el contenido de la problematización, objeción, rectificación, etc., más un refuerzo de tipo argumentativo, de tipo causal. Ese refuerzo emerge como marca colaborativa que correspondería -a nuestro juicio, y por lo que demuestran datos preliminares sobre creencias- a una representación común de lo que es estudiar en la universidad. Participar en este evento implica tomar postura y manifestarla discursivamente, incluso cuando se pregunta, se rechaza o se objeta. Con el refuerzo, el interlocutor propone, objeta, rechaza o justifica su posición, ayudando al otro a comprenderla.

Esta actitud común por parte de los interlocutores no debe entenderse como una homogeneidad grupal. Como señalan Johnson y Johnson (1999: 114):

“...cuanto más homogéneo sea un grupo, menos aportará cada miembro a los recursos grupales. Los grupos necesitan desarrollar la combinación correcta de trabajo en la tarea y habilidades sociales necesarias para hacer el trabajo. La heterogeneidad asegura una amplia variedad de recursos disponibles para el trabajo grupal".

Esta 'combinación correcta de trabajo' se estructura a partir de los aportes individuales y se hace necesaria, pues cada integrante dispone solo de una parte de la información y de un número limitado de recursos necesarios para llevar a cabo la tarea con éxito. Por lo tanto, la interdependencia positiva de recursos, roles y tareas en el desarrollo temático obliga a los miembros 
del grupo a una actitud colaborativa y a que se fortalezcan en su individualidad. Lo más importante es que cuanto mayor es la interdependencia positiva en un grupo de aprendizaje, mayor es también la posibilidad de disenso intelectual y de conflicto entre sus integrantes. Tal como afirman Johnson y Johnson (1999), cuando tales controversias son manejadas constructivamente, ellas promueven la inseguridad sobre las conclusiones propias, la búsqueda activa de mayor información, la reconceptualización del conocimiento y las propias conclusiones y, consecuentemente, confieren mayor dominio y capacidad de retención de lo que se está discutiendo.

Otro rasgo que emerge es la duplicidad funcional de muchos movimientos. Estos operan en un ámbito temático, co-construyendo un tema consensuado y, a la vez, operan en el plano propiamente interaccional: sirven para construir identidad, para reforzar a otro, para crear lazos afectivos, o como instrumentos de poder.

\section{CONCLUSIONES}

Esta investigación constituye un aporte en dos planos. En primer lugar, sus resultados entregan antecedentes sobre una práctica altamente valorada por los integrantes de la comunidad académica y que no ha sido descrita discursivamente en profundidad.

La aproximación teórico-metodológica adoptada hace posible dar cuenta de la complejidad real del fenómeno discursivo estudiado. Permite describir el dinamismo argumentativo necesario para construir conocimiento disciplinar cuando los estudiantes universitarios estudian en grupo a partir de un permanente juego de 15 movimientos estratégicos de los participantes en el evento, y permite, asimismo, percibir el valor real de ciertos movimientos aparentemente disociadores, como rechazo, objeción y rectificación.

Sería interesante que futuras investigaciones ahondaran las representaciones sociales de los participantes, estudiantes y docentes, sobre el evento Grupo de estudio. Tal investigación posibilitaría explicar con mayor claridad su engranaje y permitiría contar con una base más sólida para comprender lo que es estudiar en la universidad y el valor epistemológico que conllevan las prácticas orales en el ámbito académico.

\section{REFERENCIAS BIBLIOGRÁFICAS}

Arundale, R. (1999). An alternative model and ideology of communication for an alternative to politeness theory. Pragmatics, 9(1), 119-153.

Duarte, P. (1997). La argumentación en el intercambio conversacional. En R. Marafioti (Ed.), Temas de argumentación (pp. 73-85). Buenos Aires: Biblos. 
Gille, J. (2004). Pautas argumentativas en el diálogo espontáneo. Un estudio de conversaciones intra e interculturales. Stockholm: Stockholm University.

Harvey, A. (2006). Encuentros orales con fines de estudio. Aproximaciones al tema. En J. Falk et al. (Eds.), Discurso, interacción e identidad. Homenaje a Lars Fant (pp. 136-162). Estocolmo: Universidad de Estocolmo.

Hymes, D. (1986). Modelos de interacción entre el lenguaje y la vida social. En L. Golluscio (Ed.), Etnografía del habla: Textos fundacionales (pp. 55-89). Buenos Aires: Eudeba.

Johnson, D. \& Johnson, R. (1999). Aprender juntos y solos. Aprendizaje cooperativo, competitivo e individualista. Buenos Aires: Aique.

Koike, D. (2003). La construcción del significado en el español. Elementos pragmáticos de la interacción dialógica. En $\mathrm{D}$. Koike (Ed.), La co-construcción del significado en el español de las Américas: Acercamientos discursivos (pp. 11-24). Ottawa: Legas.

Linell, P. (1998). Approaching dialogue. Talk and interaction in dialogical perspectives. Amsterdam: John Benjamins.

Mercer, N. (1997). La construcción guiada del conocimiento. El habla de profesores y alumnos. Barcelona: Paidós.

Monereo, C. \& Durán, D. (2001). Entramats. Mètodes d'aprenentatge cooperatiu I col-laboratiu. Barcelona: Edebé.

Núñez, P. \& Oyanedel, M. (2006). El grupo de estudio: ¿Cómo co-construyen el conocimiento disciplinar los estudiantes universitarios? Ponencia. IV Encuentro Nacional de Estudios del Discurso. Voces para el discurso del mañana. Valparaíso: Pontificia Universidad Católica de Valparaíso.

Slavin, R. (1995). Cooperative learning. Massachusetts: Allyn \& Bacon.

\section{NOTAS}

1 Proyecto FONDECYT 1060566. Discurso académico: Encuentros orales con fines de estudio. 